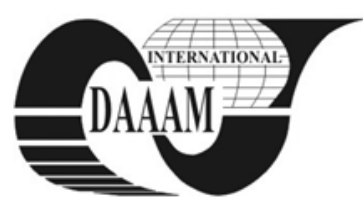

Annals of DAAAM for 2011 \& Proceedings of the 22nd International DAAAM Symposium, Volume 22, No. 1, ISSN 1726-9679 ISBN 978-3-901509-83-4, Editor B. Katalinic, Published by DAAAM International, Vienna, Austria, EU, 2011 Make Harmony between Technology and Nature, and Your Mind will Fly Free as a Bird Annals \& Proceedings of DAAAM International 2011

\title{
OPERATION ENVIRONMENT OF MOBILE ROBOTS WITH SUPERVISION CONTROL
}

\author{
ANDREEV, V[ictor] P[avlovich] \& PRYANICHNIKOV, V[alentin] E.
}

\begin{abstract}
Based on analysis of data flows and functional structure of information - measuring and control systems of mobile robots with supervisory control, proposed a combined approach to constructing such systems and multi-camera computer vision systems.
\end{abstract}

Key words: mobile robots, operation environment, computer vision systems, supervisory control, network with mobile nodes

\section{MOBILE ROBOT CONTROL SYSTEM}

Let us consider data flows in an information-measuring system of the mobile robot (MR) on the example of the operation environment shown in Fig. 1. As any complex control system, a MR involves subsystems estimating the state of the external environment and the state of the control system as well as the formation of feedback and control signals. The information-measuring system involves different sensors (data sensing devices): odometers, ultrasonic sensors, TV cameras, thermal imagers, special scanners (gamma locators), etc.

In systems with supervisory control, the data analysis and control goal formation are conducted by human beings. Therefore, the information about the environment should be delivered in a form that is customary (i.e., as a halftone color image) and one should use computer vision systems (CVSs) as the main sensor.

The signals arriving from different sensors are subjected to the influence of various noises and distortions (fluctuation noises, optical distortions, distortions caused by sensors, etc.). Thus, the system should contain a signal preprocessing unit responsible for filtering these noises and correcting the distortions. A distinguishing feature of the MR is that the incoming information has a dynamical character; consequently, the signal preprocessing unit must be real-time. Because such a huge data flow cannot be handled by an ordinary computer, the signal processing at this stage is conducted with the help of special-purpose processors.

Among signal preprocessing algorithms, the most efficient are the adaptive algorithms using the result of analyzed information about the permanently changing external environment to adjust its parameters (Andreev, 2010). The parameters of signal preprocessing can be affected also by the goal of control. The instructions for changing the preprocessing parameters arrive from an internal interface through an instruction control unit, thus making an internal control loop.

The video signals from TV-cameras and the signals from other sensors must be converted into a structured "data train" that should be prepared for transmitting through communication channels (communication environment). By a structured data train, we mean a continuous data flow from all sensors of the MR and control instructions that are ranged into a sequence in line with definite rules. The data transmission through internal chains of a MR can be performed using wired broadband digital channels of data transmission, when digital images and other data refined from different kinds of noises go directly into the multiport memory of the data-analysis unit. When a structured data train is transmitted through radio channels, the remote interface encounters a number of problems stemming from the communication environment. When analog radio channels are used, a problem of bandpass arises, which in this case should be enough for transmitting TV video signals from a set of television cameras. In addition, analog radio channels are known to have low fault-tolerance.

The transition to digital data transmission through radio channels made it possible to solve theses problems. However, an absolutely stable radio channel will never be organized; therefore, the internal interface is assigned to treat a finite set of navigation problems in an autonomous mode for the case when radio control is lost. Depending on the complexity of given problems, the data analysis and goal generation can be performed using both remote and internal interfaces.

The remote interface is responsible for converting the incoming data into a standard form, analyzing these data, generating a goal of control and corresponding instructions or a program of control, and transmitting them into the MR for execution, thus forming an external contour of control. According as the control type (remote or supervisory), the incoming data are analyzed and the control goal is generated by a human control or automation together with human. In both cases, the incoming information should be reflected on control desk monitors in a form that is most convenient for human perception. All arriving video streams must be converted into images on the screens of monitors. The latter must also involve images reflecting the indications from other MR-based sensors. The goal of control is made by a human control. Therefore, the control desk as a part of remote interface should contain control units meeting ergonomic requirements: buttons, tumbler switches, joysticks, etc. The instructions from control units are transmitted to the MR. All functions mentioned above are implemented by a control desk computer. The performance of this computer depends primarily on the number of video streams that need to be decoded and converted into a sequence of half-tone images generated with a standard frame rate $(25 / 30$ frames per second). In the case of supervisory control, this computer is assigned to perform data analysis and generation of control goal within the framework of human instructions.

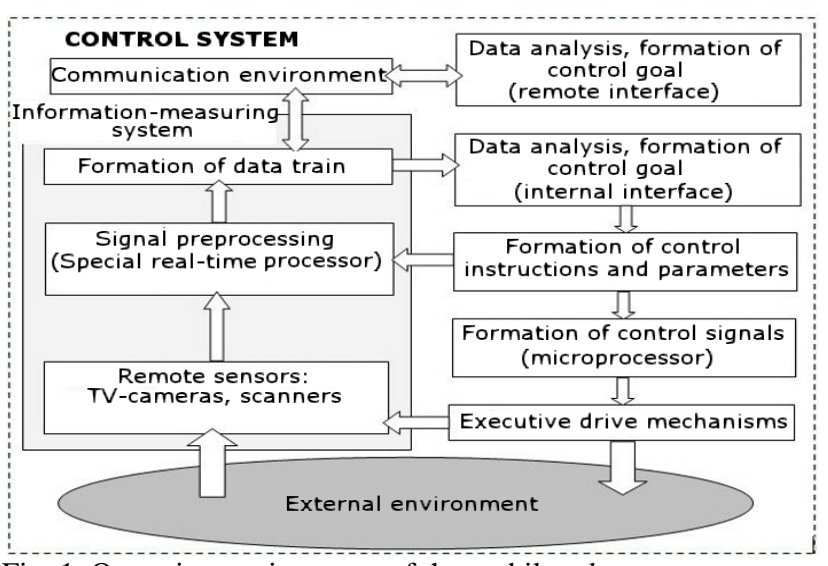

Fig. 1. Operation environment of the mobile robot 
The internal interface is responsible for converting incoming data into a standard form, analyzing data, generating a control goal (in the cases of supervisory control and loss of radio communication), and generating suitable instructions or program of control and their transfer to the MR-control instruction generating unit for execution, thus forming an internal contour of control. These functions require a computer but with modest computing resources because this requires considerable energy costs under limited energy possibilities of on-board power supply of the MR.

The unit of generation of control instructions is responsible for the transformation of decisions made from the operation of internal interface into a system of instructions determined by specific features of the unit of generation of control signals. This may include, for example, a USB to RS-232 or RS-485 converter. The unit of generation of control instructions is actually an interface transformer allowing one to use microprocessor units of different manufacturers.

The unit of generation of control signals is a transformer of control instructions into electrical signals that are sent to executive mechanisms through respective amplifiers. An example of the system of generators of control instructions and signals is a PTZ camera controller equipped with a rotary tilting mechanism and an optical scaling system.

\section{FUNCTIONAL STRUCTURE OF MR}

An analysis of data flows in the MR makes it possible to specify the required functional structure of its equipment: - a set of sensors with a corresponding system of signal generation (TV camera, thermal imager, gamma-radiation scanner, odometers, ultrasonic sensor, etc.);

- a system of data collection and filtering of signals from different sensors for the correction of distortions caused both by the fact that the gain-transfer characteristics of signal sensors are nonideal and by signal-generation;

- a system of radio channel for communication between the onboard unit and control desk;

- a repeater designed generally for acquiring a stable radio channel at large distances;

- a controller desk with a radio channel generation unit, a system for converting video signals into half-tone images (including a video data mapping control system), and a system of control bodies (for example, joysticks);

- a system of self-contained power supply for electronic units and drivers of executive mechanisms;

- for supervisory control, a computer is installed onboard MR; remote units used for making the operation of the MR control easier. These units contain controllable TV-cameras placed immediately close to the place of MR operation and allowing to observe it as an onlooker.

\section{SPECIFIC FEATURES OF CVS FOR MR}

Let us consider some key aspects of CVS construction for. Here, we take into account that the completeness and reliability of data mapping are necessary for minimizing the errors arising in the generation of control goals.

The completeness of data is ensured by the possibility of a full coverage of the locale, and the reliability of data depends on both the technical characteristics of sensors and techniques of data retrieval and representation. The technical characteristics of sensors influence on data completeness as well. It's very important for self-organizing systems (Katalinic, et al., 2002).

The full coverage of the locale on the MR is provided by the system of optical radiation sensors:

TV-camera with an optical system of omnidirectional scanning; TV-camera with a rotary tilting mechanism; several TVcameras installed onboard the MR; TV on remote units.

For MR with supervisory control, it will be most appropriate to combine the variant with a controlled multicamera CVS using TV-cameras with a rotary tilting mechanism and the possibility of optical scaling (PTZ). The remote units (RUs) must be self-contained and consequently have a suitable power-supply system and a unit of radio channel generation for communicating with the controller desk. These RUs ("satellites") can be placed on a simplified moving chassis (on the robot-observer).

The use of multi-camera CVSs brings up the question of multi-stream video transfer through a radio channel. One of the traditional answers is to use a broadband analog radio channel. The use of IP-cameras with hardware compression of images makes it possible to solve the problem of multi-stream video more cheaply and reliably. In this case, analog-digital conversion occurs making it possible to use the most advanced Ethernet-based methods. Then, the problem is reduced to the creation of a local computing network with mobile nodes represented by $\mathrm{TV}$-cameras installed on onboard units and remote units as well as, on the one hand, an onboard computer and, on the other hand, a computer on the controller desk (Andreev et al., 2010). This CVS has the following properties. 1. Multi-stream video: video signals can be transferred simultaneously from several TV-cameras without reducing the quality of images, which is achieved by using highly efficient compression algorithms for the sequence of frames. 2. Uniting CVS elements through digital radio channels: the Wi-Fi or WiMax standards are used. 3. Scalability: the possibility of easy upgrading of any MR resources (IP-remote units). 4. Highquality images: the possibility of using high-resolution TVcameras with good color rendering. 5. Digital systems of image processing: a special digital processors and versatile computers. 6. Enhanced noise protection: digital channels of data transmission prevent video signals from distortion. 7. Functionality: digital channels make it possible to transfer not only video but also audio signals and control signals. 8. Distributivity: computing resources can be distributed between MR units. 9. Multiuser mode: the possibility retrieval of data on several controller desks (including those connected to the system via the Internet). 10. Control via the Internet: the possibility of analyzing the operation of MR and controlling over its executive mechanisms at any distances.

\section{CONCLUSION}

Within the frameworks of the concept and under contracts signed with different agencies and universities, our laboratory has developed AMUR-series mobile robots and CVSs installed on heavy-duty mobile robots (Brokk-110D, Brokk-330). These systems proved their efficiency, including for conditions of operating in emergency situations MR (Andreev et al., 2009).

\section{REFERENCES}

Andreev V., Kirsanov K., Pronkin V., Pryanichnikov V., Prysev E. (2009). Computer vision for mobile robot on the base of Wi-Fi. // Intelligent and Adaptive Robots: Radiotechnika, ISSN 2070-0814,No1,2, pp.49-63, Moscow

Andreev V., Pryanichnikov V., Prysev E. (2010). Multi-access control of distributed mobile robotic systems on the base of networking technologies. Annals of DAAAM for 2010\&Proceedings of the 21st International DAAAM Symposium, ISSN 1726-9679, pp.15-16, Zadar, Croatia

Andreev V. (2010). Video Signal Correction for Scanning Photocell Array in the IR-Computer Vision Systems. Annals of DAAAM for 2010\&Proceedings of the 21st International DAAAM Symposium, ISSN 1726-9679, pp.17-18, Zadar, Croatia

Katalinic, B., Kordic, V. (2002) Concept of Design and Scheduling of Self-Organizing Complex Flexible Assembly System, Proceedings of the 4th International Workshop on Emergent Synthesis - IWES 02, ISBN 961-4238-49-3, pp. 89-96, March 12-13, 2002, Kobe, Japan 